\title{
Manuskriptfassung zu:
}

Ott, Christine (2016): Geschlechterstereotypen auf der Spur. Ein Plädoyer für mehr Linguistik in der Bildungsforschung, in: Groppe, Carola/Kluchert, Gerhard/Matthes, Eva (Hrsg.): Bildung und Differenz. Historische Analysen zu einem aktuellen Problem. Wiesbaden: Springer VS, S. 161-183.

\section{CSpringerVS}


Christine Ott

Geschlechterstereotypen auf der Spur

Ein Plädoyer für mehr Linguistik in der Bildungsforschung

\section{Einleitung}

Die Sprachwissenschaft ist nicht nur jene Wissenschaftsdisziplin, die sich mit Satzbauplänen oder Flexionsendungen beschäftigt; sie erforscht Sprache zunehmend in ihren kulturellen Bezügen und untersucht den Zusammenhang von Sprache, Denken, Wissen. Die Genderlinguistik arbeitet dabei an einem Beschreibungssystem für sprachliche Konstruktionen von Geschlechterkonzepten (vgl. Spieß 2012). Für zahlreiche LinguistInnen - wie auch für die Soziologen Berger und Luckmann (1977, S. 72 f.) - stellt Sprache das zentrale Symbolsystem dar, durch welches unter anderem Geschlechtervorstellungen hervorgebracht werden.

Das methodische Werkzeug, mit dem der Anteil der Sprache an der Konstruktion von Geschlechter,realitäten' sichtbar gemacht werden kann, stellt die Linguistik bereit. Auch bei sozial- und erziehungswissenschaftlichen Fragestellungen lohnt ein Einbezug linguistischer Analyseverfahren und -kategorien. Dieser Aufsatz zeigt Perspektiven für den interdisziplinären Austausch und Fachwissenexport am Beispiel des Bildungsmediums Schulbuch und der Identitätskategorie Geschlecht auf. Als wichtiger Baustein der schulischen Sozialisation partizipieren Schulbücher auch am Aufbau von Männlichkeits- und Weiblichkeitskonzepten. Ihr Status als Leitmedium ${ }^{1}$ macht Schulbücher $\mathrm{zu}$ einem besonderes relevanten Untersuchungsgegenstand, zumal, wenn die Inhalte als legitimiert gelten dürfen, d. h. die Bücher ein Zulassungs- und Begutachtungsverfahren erfolgreich durchlaufen haben. ${ }^{2}$ Sie vertreten und transportieren $^{3}$ einen von bildungspolitischer Seite wenigstens nicht abgelehnten Entwurf einer sozialen Wirklichkeit. Welche Weltdeutung legen Schulbücher dann potenziell nahe? Bisherige Schulbuchanalysen haben kaum herausgearbeitet, welche Rolle der Sprache hierbei zukommt. Im Zuge der folgenden Analyse wird beleuchtet, inwiefern Sprache bzw. ihre ProduzentInnen eine Differenzsetzung in ,weiblich ${ }^{\star}$ - ,männlich ${ }^{\star}$ aktualisieren sowie einem essentialistischen Zwei-Geschlechter-Modell und naturalistischen Vorstellungen von ,typisch weiblichen" oder ,typisch männlichen“ Eigenschaften wie Verhaltensweisen Vorschub leisten. Die Diskurstheorie Foucaults in Butler'scher Interpretation bildet den theoretischen Bezugsrahmen. Ich verstehe dabei Diskursanalyse nicht als Methode, sondern als analytische Perspektive, aus der heraus

\footnotetext{
${ }^{1}$ Unter anderem für das Fach Mathematik bescheinigte die Lehrerbefragung im Forschungsprojekt „Bildungsmedien Online“ der Universität Augsburg 2013 den hohen Stellenwert des Schulbuchs gegenüber anderen Medien und Unterrichtsmaterialien (vgl. Verband Bildungsmedien 2013; vgl. auch Neumann 2015). Neuere fachspezifische empirische Studien zum Einsatz von Schulbüchern im Unterricht sind zu Deutschbüchern bei Ballis und Gaebert (2012), zu Geschichtslehrbüchern bei Brieske (2007) verzeichnet.

${ }^{2}$ Die Stadtstaaten Berlin und Hamburg sowie das Saarland und Schleswig-Holstein verzichten auf ein eigenes zentrales Zulassungs- und Prüfverfahren; für weitere Informationen zur Zulassung von Schulbüchern in Deutschland vgl. Stöber (2010), Wendt (2010), Leppek (2002).

3 Mit ,vertreten und transportieren" und weiteren ähnlichen Formulierungen verweise ich auf die „Doppelgesichtigkeit“ des Diskurses: Wie Warnke (2013, S. 102) ausführt, funktionieren Zeichen in der Kommunikationspraxis zwar durchaus repräsentational, d. h. einer Abbildfunktion von Sprache wird wenigstens in der Alltagssprache vertraut. Erst in diachroner Betrachtung aber wird die Konstruiertheit dieser Repräsentationalität als Ergebnis von Konventionalisierungsprozessen erkennbar und kann jede Äußerung als teilhaftig an der Konstruktion und (Re-)Produktion von Wissen betrachtet werden.
} 
gefragt wird, was die Voraussetzungen für das Auftauchen und Verstehen von Aussagen zu den Geschlechtern und zu Geschlechtlichkeit sind, was also nicht (zwingend) explizit gemacht wird, aber Bedingung für das Hervorbringen sowie Verstehen von Sprache ist und gewissermaßen stillschweigend vorausgesetzt wird.

Das Rahmenthema „Bildung und Differenz“ wird im Folgenden vorwiegend diskursgeschichtlich perspektivert. Der Beitrag beschreibt einerseits, wie Geschlechterkonzepte in der Beispieltextsorte Schulbuch sprachanalytisch ermittelt werden können. Er bindet die Frage nach Geschlechterstereotypie an das konkrete Sprachmaterial zurück. Mit der linguistischen Analyse der Schulbücher sollen Einsichten in die sprachliche Verfasstheit von Vorstellungen über Geschlechter gegeben werden. Eine Analyse der Sprache will somit offenlegen, welche sprachlichen Einheiten auf welche Weise an der Konstruktion von gegebenenfalls stereotypen Geschlechterkonzepten mitwirken. Ein Untersuchungsschema für Textanalysen zu Geschlechterkonzepten wird in Auszügen vorgestellt. Es wurde im Rahmen meiner Dissertation an Schulbüchern entwickelt und ist in seiner Anlage auch für andere Textsorten geeignet. Im analytischen Vorgehen unterscheidet sich diese Untersuchung von älteren Schulbuchanalysen zu Rollenbildern (vgl. z. B. Lopatecki und Lüking 1989; Analysen im Überblick bei Fichera 1996 und Hunze 2003) unter anderem und vor allem darin, dass Stereotype nicht zum Ausgangspunkt der Analyse gemacht werden - was womöglich den Blick für Unerwartetes oder Widersprüchliches versperrt -, sondern Stereotype erst bei der Interpretation des Datenmaterials und der Auswertungen herangezogen werden. Bei der Beschreibung des Schemas wird auf erhebungspraktische Probleme und Differenzierungsmöglichkeiten im Umgang mit Sprachdaten eingegangen, die auch in inhaltsanalytischen Untersuchungen, z. B. nach Mayring, berücksichtigt werden sollten. Im Anschluss werden Untersuchungsergebnisse aus der Analyse von zeitgenössischen und historischen Schulbüchern präsentiert. Das Untersuchungskorpus besteht hierbei ausschließlich aus Lernmitteln für den Rechen-/Mathematikunterricht. Die Entscheidung für diese Bücher liegt im Fach begründet: Im Unterschied zu den Geistes- und Sozialwissenschaften gilt die Mathematik weithin als ideologisch unbestechlich und in besonderem Maß sachorientiert.

Andererseits berücksichtige ich in diesem Beitrag den unmittelbaren Entstehungskontext von Schulbüchern und stelle Ergebnisse einer Untersuchung der Ebene der AkteurInnen d. h. von Personen und Institutionen, die an der Konzeption, Erstellung und Zulassung von Bildungsmedien beteiligt sind, zum Stellenwert von Gender Mainstreaming und Geschlechtergerechtigkeit in der Schulbucharbeit und Zulassungspraxis vor. Diese Untersuchung steuert nötiges Hintergrundwissen für die Interpretation von Veränderungen im Sprachmaterial der Schulbücher bei und zeigt, inwiefern die Geschlechterthematik und gendertheoretische Fragen im bildungssystemischen Entstehungszusammenhang mitreflektiert werden.

\section{Geschlechtersensibilität in der Schulbucharbeit und Schulbuch-Zulassungspraxis}

SchulbuchautorInnen, -herausgeberInnen, -redakteurInnen sowie Zulassungsstellen und GutachterInnen - in der Regel Lehrkräfte, die im Auftrag der Zulassungsstelle Schulbücher bewerten - wurden als relevante unmittelbare Instanzen identifiziert, die auf die inhaltliche und sprachliche Gestaltung von Schulbüchern Einfluss nehmen (vgl. auch Höhne 2003; weiterführend: Lässig 2009). Zwischen Oktober 2013 und Februar 2014 habe ich 21 ExpertInnen aus den genannten Kreisen an AkteurInnen in leitfadengestützten Interviews zur Relevanz von 
Genderfragen in der Konzeption und Zulassung von Schulbüchern befragt. Die SchulbuchautorInnen und -herausgeberInnen stellten mit 9 Befragten die größte Gruppe. Für jede Interviewgruppe - 1) AutorInnen und HerausgeberInnen, 2) RedakteurInnen, 3) GutachterInnen, 4) MitarbeiterInnen der Zulassungsstelle - wurde ein Leitfaden ausgearbeitet, der neben biographischen Fragen an die interviewte Person (z. B. wie häufig sie als GutachterIn tätig war und wie sie zu dieser Tätigkeit gekommen ist) jeweils in unterschiedlicher Ausführlichkeit und Fragerichtung folgende Themenkomplexe beinhaltete: ${ }^{4}$

- Inhalte und Form behördlicher Vorgaben in Vergangenheit und Gegenwart, die das Schulbuch im Allgemeinen und die Geschlechterthematik im Besonderen betreffen

- Schulbuchgutachten in Vergangenheit und Gegenwart und ihre Bezugnahme auf die Geschlechterthematik

- Inhalte und Form verlagsinterner und redaktioneller Vorgaben in Vergangenheit und Gegenwart zum Umgang mit der Geschlechterthematik

- Vergleich des Stellenwerts der Geschlechterthematik in der Zulassungspraxis oder Schulbuchkonzeption vor allem nach Fach und Bundesland

- Kommunikation zwischen Verlag/AutorInnen oder GutachterInnen und Behörde (Ansprechpersonen, Kommunikationswege, Zulassungs- und Prüfvorgang, Diskussionspunkte, Dokumentation des Prüfvorgangs), Struktur der Behörde

Die Ergebnisse der Befragung sind nicht repräsentativ, bieten aber Einblicke in eine Praxis, die der Forschung in dieser Form noch nicht zugänglich war. In den Interviews stand im Mittelpunkt, welche konkreten inhaltlichen und sprachlichen Feinheiten mit Blick auf die Geschlechterthematik beim Erstellen von Schulbüchern berücksichtigt werden, u. a. weil sie im behördlichen Genehmigungsverfahren der Schulbücher eine Rolle spielen.

Häufig existieren von einem Lehrwerk mehrere Länderausgaben. Wo sich Lehrpläne und Rahmenrichtlinien verschiedener Länder ähneln, streben Verlage zwar aus wirtschaftlichen Gründen die Zulassung einer Lehrwerksausgabe in mehreren Ländern an. Allerdings ist für den Zielmarkt Bayern beispielsweise meist eine eigene Ausgabe nötig. Was am Ende in den für den Unterricht zugelassenen Schulbüchern zu lesen ist, wird von den EntscheidungsträgerInnen normalerweise das jeweilige Kultusministerium oder Landesschulinstitut - gebilligt oder zumindest nicht zurückgewiesen. Unter anderem in dieser Hinsicht können Schulbücher als kultur- und gesellschaftsabhängige Zeitdokumente verstanden werden, die ein zeitgebundenes Weltbild vertreten und bestimmte soziale Praktiken oder Rollen als Norm ausweisen.

Seit Beginn der feministischen Schulbuchkritik ab Ende der 1960er Jahre war es stark vom Bundesland, in dem ein Schulbuch erscheinen sollte, abhängig, ob und in welchem Maß Forderungen nach mehr Geschlechtergerechtigkeit und nach einem vor allem differenzierteren Frauenbild im Zulassungsverfahren berücksichtigt wurden. In den meisten Bundesländern, z. B. in den beiden bevölkerungsreichsten, dabei bildungspolitisch differierenden Ländern NordrheinWestfalen und Bayern, müssen Schulbücher für Kernfächer wie Mathematik oder Deutsch bis einschließlich der Sekundarstufe I ein Begutachtungsverfahren durchlaufen. Meist prüfen zwei GutachterInnen ein Schulbuch auf seine Tauglichkeit, d. h. seine Lehrplankonformität, didaktische und fachwissenschaftliche Angemessenheit sowie seine Übereinstimmung mit

\footnotetext{
${ }^{4}$ Die Leitfäden und Interview-Mitschriften sind bislang unveröffentlicht. Tonaufnahmen der Gespräche waren von der Mehrzahl der Befragten abgelehnt worden, weswegen keine wortgetreuen Transkriptionen der Interviews angefertigt werden konnten.
} 
geltenden Rechtsnormen, und empfehlen am Ende die Zulassung oder aber die Ablehnung. Die Zulassungsstelle entscheidet dann, ob sie sich dem Urteil der GutachterInnen anschließt.

Ministerialbeamte und SchulbuchmacherInnen wiesen in den Gesprächen nun darauf hin, dass mindestens seit Mitte der 1990er Jahre das Prinzip Geschlechtergerechtigkeit zu den Zulassungskriterien zählt und bereits zuvor mehr oder weniger ausgeprägt - je nach politischer Couleur der Landesregierung - in Gutachten auf die Geschlechtersensibilität eines Schulbuchs kritisch eingegangen wurde. Gerade $\mathrm{zu}$ Beginn der feministischen Bewegung und Schul(buch)kritik gingen Bestrebungen zu mehr Geschlechtergerechtigkeit von sensibilisierten Schulbuchautorinnen aus, ein wenig zeitversetzt wurde ab Ende der 1970er Jahre von der nordrhein-westfälischen und der hessischen Zulassungsstelle bzw. von deren GutachterInnen Kritik an stereotypen Geschlechterdarstellungen und der sprachlichen Diskriminierung von Frauen geübt. ${ }^{5}$ Diese Erfahrungen bewegten manche Verlagsredaktionen dazu, neue Standards im Umgang mit dem Thema zu etablieren und diese auch in Ausgaben für andere Länder umzusetzen, von denen solche Kritik bislang nicht geübt worden war. Sie kamen damit - auch aus wirtschaftlichen Gründen - einer ohnehin absehbaren Entwicklung zuvor und arbeiteten dabei - wissentlich oder nicht - aktiv an einer Vereinheitlichung von GeschlechtersensibilitätsStandards mit.

Ein Blick in die aktuell gültigen Verordnungen und Kriterienkataloge der Zulassungsbehörden sowie in die Schulgesetze der Länder zeigt, dass - zumindest in der Theorie Geschlechtergerechtigkeit gegenwärtig länder- und auch fächerübergreifend zu den Zulassungskriterien für Schulbücher zählt. So führt der bayerische allgemeine Kriterienkatalog für die Zulassung von Lernmitteln unter dem Punkt „Gleichberechtigung“ mit Referenz auf den Gleichheitsgrundsatz im Grundgesetz der Bundesrepublik Deutschland sowie in der Bayerischen Verfassung Folgendes aus:

Es ist darauf zu achten, dass Männer und Frauen gleichberechtigt dargestellt sind [...]. Die Lebenswirklichkeit von Frauen in unserer Gesellschaft sowohl im Hinblick auf Belastungen und Konflikte wie auch hinsichtlich ihrer Teilnahme am Berufsleben und am öffentlichen Leben muss ausreichend dargestellt werden. Das Lernmittel darf nicht der Entwicklung einseitiger Vorstellungen über die Position von Männern und Frauen in Gesellschaft und Familie oder anderen Formen des Zusammenlebens Vorschub leisten. (StMUKWK 2014, S. 8 f.)

SchulbuchmacherInnen bestätigten in den Interviews, dass heute kaum mehr Unterschiede nach Bundesländern und Fach in der Zulassungspraxis hinsichtlich des Kriteriums Geschlechtergerechtigkeit feststellbar seien, zudem Geschlechtersensibilität im Schulbuch ganz selbstverständlich und in allen Fächern gleichermaßen angestrebt werde. Häufig sind den RedakteurInnen die behördlichen Bestimmungen der Länder im Einzelnen nicht bekannt, wie in den Interviews deutlich wurde. Prinzipiell werde im Verlag und AutorInnenteam mit Blick auf Geschlechtersensibilität aber geachtet auf:

\footnotetext{
${ }^{5}$ In diesem Zusammenhang sei auf das Treffen von WissenschaftlerInnen, Verlagen und politischen AkteurInnen hingewiesen, das bereits 1972 am Internationalen Schulbuchinstitut Braunschweig stattgefunden hat und bei dem als Ergebnis festgehalten wurde, die Geschlechterdarstellung im Schulbuch zeitgemäßer und gleichberechtigt zu gestalten; Mathematikbücher allerdings wurden im Ergebnispapier nicht als änderungsrelevant genannt (vgl. Stein 1979, S. 295 ff.).
} 
- Vermeiden stereotyper Situationen und Berufszuweisungen (z. B. im Mathematikbuch: Haushaltsgeräte nicht nur von Frauen kaufen lassen)

- keine geschlechterstereotypen Illustrationen und Themen (Negativbeispiel: Sportliche Aktivitäten werden in Bild und Text von Jungen ausgeübt)

- ausgewogene Verteilung von richtigen und falschen Lösungsangeboten durch weibliche und männliche Schulbuchfiguren

- geschlechtersensible Aufgabenformulierungen, z. B. Schülerinnen und Schüler

- paritätisches Verhältnis von Bezeichnungen für Mädchen/Frauen und Jungen/Männer

- gleichmäßige Verteilung von HandlungsträgerInnenschaft auf die Geschlechter

- historische Leistungen von Frauen gleichermaßen wie jene von Männern würdigen (Maßgabe richtet sich vor allem auf Geschichtslehrbücher)

- möglichst ausgewogenes Verhältnis von Frauen und Männern im AutorInnenteam (was im Fach Mathematik ab der Sekundarstufe I allerdings selten eingelöst wird, soweit sei vorgegriffen)

Diese Kriterien waren in der Vergangenheit als Kritik in Gutachten aufgetaucht und aus dieser Erfahrung heraus zu einer Art Checkliste gesampelt worden, die als solche allerdings in keiner der befragten Redaktionen explizit fixiert existiert, sondern als selbstverständlicher Standard gilt. Wir werden bei der Analyse der Schulbücher auf einzelne Punkte zurückkommen.

Zudem waren sich die befragten Verlage und vor allem HerausgeberInnen von Lehrwerken einig, dass Gutachten regelhaft auf das Kriterium Geschlechtergerechtigkeit eingehen würden. Dies hat sich allerdings beim direkten Blick in die zugänglich gemachten Gutachten nicht bestätigt. Selten findet sich darin eine ausführliche Bemerkung; am ehesten noch wird der Punkt mit „Rollenklischees wurden vermieden“ oder „Auf eine gleichwertige Darstellung der Geschlechter wurde geachtet" abgehandelt. Es bleibt bei der anschließenden Schulbuchanalyse abzuwarten, ob die Abwesenheit einer Stellungnahme als fehlende Sensibilität oder ein Geringschätzen von nicht-fachdidaktischen und -wissenschaftlichen Beurteilungsaspekten zu werten ist oder als Hinweis darauf, dass es nichts zu beanstanden gibt.

Ein grundlegender Diskussionspunkt in den Redaktionen und AutorInnenteams ist laut Interviews, ob sich bei der Darstellung der Geschlechter im Schulbuch eher an einer sogenannten gesellschaftlichen Realität (das hieße für Deutschland z. B. eine Unterrepräsentanz von Frauen in Führungspositionen und in MINT-Berufen) oder an einem gesellschaftlichen Gleichheitsideal orientiert werden soll. Im angeführten Zitat aus dem Kriterienkatalog wird darauf nicht eingegangen, beide Auslegungen sind möglich. So ist es den einzelnen Teams überlassen, ob sie eine - wie auch immer fassbare - gesellschaftliche Realität als Ausgangspunkt nehmen oder dem konstruktivistischen Potenzial von Schulbüchern Rechnung tragen. Das Problem taucht auch bei der Frage auf, auf welche Weise sogenannte geschlechtsspezifische Vorlieben in die Schulbuchwelt integriert werden: Ein Schulbuch habe für Mädchen und Jungen jeweils spezifische Identifikationsangebote zu schaffen, zum Beispiel das Rahmenthema Fußball für die Jungen und im Bereich Sport Schlittschuhlaufen oder Ballett für die Mädchen anzubieten (doing difference, doing gender), merkten SchulbuchmacherInnen unterschiedlicher Fachbereiche an. Dagegen verfolgt einer der befragten Schulbuchverlage nach eigener Auskunft das Prinzip des Gender Mainstreaming auch auf der Ebene der Schulbucharbeit derart, dass die Geschlechter stets gleichberechtigt und gleichbefähigt dargestellt und gegenwärtig vielleicht existente Differenzen in der Freizeitgestaltung und den Vorlieben gerade nicht aktualisiert und relevant gesetzt werden sollen (undoing gender); auf die beiden Beispiel-Themen angewandt, würde das 
bedeuten, dass sowohl Mädchen als auch Jungen im Schulbuch Fußball spielen oder Schlittschuh laufen.

Ein weiterer Befund aus den Interviews erscheint erwähnenswert: Unabhängig voneinander wiesen drei Interviewpartner, die jeweils über Jahrzehnte in der Schulbucharbeit tätig waren, darauf hin, dass geschlechtersensible Sprache im Sinn einer Gleichbehandlung oder Neutralisierung von Geschlecht in der Sprache (vgl. Hellinger und Bierbach 1993; radikalisiert bei Hornscheidt 2012) nicht mehr gleichermaßen konsequent wie noch vor 10 bis 15 Jahren verwendet werde. Es reiche zunehmend aus, den Gesslerhut ab und an zu grüßen, wie es in einem Interview mit SchulbuchredakteurInnen formuliert wurde, was so viel bedeutet wie, guten Willen zu zeigen, indem gelegentlich geschlechtersensible Formen eingestreut werden. Denn aktuell sei ein umsichtiger Umgang mit den Themen Inklusion und Migration im Zulassungsverfahren wichtiger geworden. Ein Indiz dafür, dass Schulbuchwissen Konjunkturen unterliegt und das Prinzip der Gleichbehandlung der Geschlechter, mit Foucault gesprochen, (noch) nicht ins Archiv übergegangen ist?

Als Fazit der Interviewauswertung ist in jedem Fall festzuhalten, dass in Verlagen und unter SchulbuchautorInnen ein erhöhtes Bewusstsein für das Thema Geschlechtergerechtigkeit besteht. Doch scheint es entscheidend von der einzelnen Person abhängig - unter den Befragten beispielsweise von der gendertheoretisch versierten Redakteurin oder der feministisch engagierten Herausgeberin -, in welcher Konsequenz, in welcher Detailliertheit und nach welcher theoretischen Prämisse (doing vs. undoing gender) Geschlechtersensibilität umzusetzen verfolgt wird oder aus Sicht der GutachterInnen als relevantes Bewertungskriterium angelegt wird. Dies zeigt unter anderem auch, welch großen Spielraum die Zulassungsstellen bei der Umsetzung der Gleichberechtigungsforderung tatsächlich einräumen.

Im Folgenden wird untersucht, ob der in den Interviews beschriebene Standard für geschlechtersensible Schulbücher für das Fach Mathematik gegenwärtig umgesetzt ist. Hierfür habe ich 12 Mathematikbücher für die 7. Jahrgangsstufe ausgewählt, die entweder im eher konservativen Bayern aktuell zugelassen sind und auch tatsächlich eingesetzt werden oder aber in den als bildungspolitisch fortschrittlich geltenden Ländern Nordrhein-Westfalen und Hessen. ${ }^{6}$ Die Ergebnisse der Analyse der zeitgenössischen Bücher werden mit linguistischen Auswertungen von je zwei historischen Siebtklass-Rechenbüchern der wilhelminischen Kaiserzeit (1890er Jahre) und der Adenauerzeit (1950er Jahre) verglichen, um Unterschiede oder auch Kontinuitäten besser erkennen und beschreiben zu können. Die historischen Rechenbücher wurden zufällig aus dem umfassenderen Schulbuchkorpus meines Forschungsprojekts mit derzeit 88 Mathematik- und Sprachlehrbüchern aus den letzten 140 Jahren ausgewählt. Es handelt sich bei den vier historischen Rechenbücher um Volksschulbücher, die in zahlreichen Auflagen erschienen sind, was auf einen hohen Verbreitungs- und Nutzungsgrad schließen lässt, und/oder von bekannten MathematikerInnen (wie Johann Friedrich Heuner) oder erfolgreichen SchulbuchautorInnen (wie Michael Hagen) verfasst wurden. Zunächst stelle ich das Untersuchungsschema vor, mit dem die Bücher auf Geschlechterkonzepte analysiert werden.

\footnotetext{
${ }^{6}$ Über stichprobenhafte Nachfragen an Schulen und bei Verlagen wurde ermittelt, welche Mathematikbücher an den verschiedenen Schularten im Schuljahr 2013/14 sowie in den unmittelbar vorausgehenden Jahren überwiegend im Gebrauch waren. Das Untersuchungskorpus richtet sich in der Zusammensetzung in der Variable Schulart tendenziell nach den Schulbesuchszahlen; aus diesem Grund sind zum Beispiel an zeitgenössischen Schulbüchern mehr Gymnasial- als Hauptschulbücher im Korpus.
} 


\section{Methodisch-empirisches Geschlechterkonzepten}

Schulbücher weisen je nach Schulfach eine spezifische Struktur bzw. Architektur auf (vgl. Heer 2011), so dass für manche Fächergruppen einige Analyseverfahren geeigneter sind als für andere. Sozialkundebücher beispielsweise können gewinnbringend auf Argumentationsmuster untersucht werden, sofern das Thema Geschlechtergerechtigkeit darin - z. B. im Zusammenhang mit dem Grundgesetz - behandelt wird (vgl. Ott 2014b). Das in diesem Beitrag vorgestellte Verfahren macht es nun gerade nicht erforderlich, dass ein Text das Untersuchungsthema explizit zum Gegenstand hat. Mit dem Untersuchungsschema kann subtiles Wissen über die Geschlechter erhoben werden, wie es Beispielsätzen zu grammatischen Phänomenen im Deutsch- und Fremdsprachbuch oder Sachaufgaben für den Mathematikunterricht mitgegeben ist. In einer Untersuchung zu Geschlechterkonzepten im Mathematikbuch sind somit die Rahmungen der Rechenaufgaben, „die Einkleidungen der Mathematik in einen Alltagskontext“ (Postupa und Weth 2011, S. 165), von Interesse. Mittels Sprache wird der situative Rahmen aufgespannt, den die SchulbuchmacherInnen weitgehend frei wählen können. Ich gehe davon aus, dass in solchen Rahmungen meist auf Situationen zurückgegriffen wird, die als unmarkierter Standard gelten oder gelten sollten. Welche Standards werden also gewählt, welche Geschlechterkonzeption wird bei der Abfassung der Aufgabenstellungen zugrunde gelegt und konstituiert? Was gilt den GutachterInnen und Zulassungsstellen als akzeptabel oder unauffällig und welche Veränderungen lassen sich in der Ausgestaltung der Rahmensituationen feststellen?

Das aufgestellte Untersuchungsschema zur Analyse von Schulbüchern erfasst Phänomene auf drei sprachlichen Ebenen: Es bewegt sich auf der Ebene einzelner Wörter, wendet sich Einheiten auf der Ebene von Aussagen zu und berücksichtigt auch Zeichen auf Textebene.

Auf der Wortebene werden vor allem Personenbezeichnungen und Pronomen, die auf Personen referieren, analysiert, im Folgenden Personenreferenzformen genannt. Entsprechend ihren grammatischen Eigenschaften werden sie Kategorien wie Numerus, Genus, Wortart, Wortbildungstyp zugewiesen und semantische Eigenschaften im Besonderen erhoben, unter anderem über eine Kodierung als Berufsbezeichnung (der Fall bei Lehrerin, Kaufmann), Verwandtschaftsbezeichnung (der Fall bei Tante, Bruder) oder Eigenname (Vor- und Nachnamen).

Die Zuweisung von Personenreferenzformen zu einem Geschlecht ist in Analysen von Geschlechterkonzepten mit die wichtigste vorzunehmende Kodierung. Beinahe alle Auswertungen am erhobenen Sprachmaterial bedienen sich der Kategorie Geschlecht als Variable. Dass eindeutige Zuweisungen allerdings nicht immer möglich sind und eine intensivere Auseinandersetzung mit dem konkreten Sprachmaterial erforderlich ist, wurde in Schulbuchstudien mit verwandtem Forschungsinteresse nicht als Erhebungsproblem thematisiert (am ehesten davon auszunehmen: Schärer 2008). Dabei ist bei zahlreichen Personenreferenzformen mit dem Genus Maskulinum zu beachten, dass diese auch ,generisch", d. h. gemischtgeschlechtlich oder geschlechtsabstrahierend, verwendet sein können und erst die textliche Umgebung im Schulbuch Aufschluss darüber gibt, ob eine Referenz auf eine männliche Person vorliegt oder ob gemischtgeschlechtlich oder geschlechtsabstrahierend referiert wird. Normalerweise gilt bei Personenreferenzformen im Deutschen: Wenn ein solcher Ausdruck ein feminines Genus hat, dann referiert der Ausdruck konventionell auf eine oder mehrere weibliche Personen, und wenn er maskulin ist, dann referiert er konventionell auf eine oder mehrere männliche Personen. Das generische Maskulinum ist eine Sprachkonvention, das diesen 
sogenannten Genus-Sexus-Zusammenhang bei den Personenreferenzformen unterläuft. ${ }^{7}$ Die Datenaufnahme kann daher nicht einfach derart vonstatten gehen, eine maskuline Personenreferenzform in jedem Fall als eine Referenz auf eine männliche Person zu werten und zu kodieren. Ein Schüler kann - sofern nicht beispielsweise durch einen Vornamen, der konventionell für Männer/Jungen verwendet wird, vereindeutigt - auch irgendeine Person meinen, welchen Geschlechts auch immer. Bei der Datenerhebung muss nicht-eindeutig referierenden Maskulina mit einem ausdifferenzierten Kodiersystem Rechnung getragen werden. Es sind daher neben ,männlicher Referenz' und ,generischer Referenz' weitere Kodierungsmöglichkeiten anzubieten.

Eine verbreitete Strategie, geschlechtersensibel $\mathrm{zu}$ formulieren, ist die Verwendung von Paarformeln, z. B. Schülerinnen und Schüler. ${ }^{8}$ Solche und weitere Bi- oder auch Multinomiale kommen in den Schulbüchern häufiger vor: ein Schüler oder eine Schülerin, Männer und Frauen oder Klaus, Peter und Tina. Sie lohnen eine eingehendere Untersuchung und können beispielsweise auf Abfolgemuster hin befragt werden. Von Interesse ist dabei, ob sich stabile Abfolgen und Abfolgeprinzipien finden, die nicht allein durch formale (z. B. klangliche) Prinzipien erklärt werden können. Kommen Personenreferenzformen für männliche Personen nun vielleicht deutlich häufiger an erster Stelle vor, was als Ausdruck einer male bias gelten könnte, wenn mit Müller (1997) angenommen wird, dass in stabilen Reihungen Wichtigeres und Typischeres voransteht? Der Mann als Normmensch und Prototyp eines Menschen also? Welche Schlüsse sind daraus $\mathrm{zu}$ ziehen, wenn in bestimmten Abfolgen von Verwandtschaftsbezeichnungen gegebenenfalls wiederum Bezeichnungen wie Mutter eher erstpositioniert werden? Bereits auf der Ebene der Personenreferenzformen lassen sich relevante Interpretationslinien entwickeln, die allerdings erst durch weitere Analysen einen belastbaren interpretativen Befund ergeben. Darüber hinaus mag es bei der Untersuchung der Schulbuchsprache, wie der Untersuchung solcher Reihungen, sinnvoll sein, umfangreichere Textkorpora einzubeziehen. ${ }^{9}$ So können Muster im Schulbuch auf ihr Vorkommen in anderen sprachlichen und thematischen Zusammenhängen überprüft und die Ergebnisse von Schulbuchanalysen auf breiterer Basis eingeordnet werden.

Neben den Personenreferenzformen selbst bietet die unmittelbare Textumgebung aufschlussreiche Analysekategorien. Wie sind die Ausdrücke beispielsweise attribuiert? Mit welchen Verben kommen sie gemeinsam vor? Deren Grobklassifizierung in Handlungs-, Vorgangs- und Zustandsverben ermöglicht zusammen mit der Bestimmung der semantischen Rollen der Personenreferenzformen einen differenzierten Zugriff auf die Standardfrage von Schulbuchanalysen zum Aspekt Geschlecht: jene nach der HandlungsträgerInnenschaft. Für eine

\footnotetext{
${ }^{7}$ Weitere Ausnahmen vom sogenannten Genus-Sexus-Zusammenhang sind beispielsweise Diminutivbildungen, wie Mädchen, Kerlchen, substantivierte Adjektive und Partizipien im Plural (die Angestellten) und Lexeme mit einem Genus Epikoinon, d. h. einem Genus, das nichts über das Geschlecht der bezeichneten Person aussagt (u. a. das Kind, die Person, der Mensch). Die Menge der Lexeme, die nicht durch Diminutivbildung oder Konversion entstanden sind und vom sogenannten Genus-Sexus-Zusammenhang abweichen, fällt für das Deutsche insgesamt klein aus (vgl. Schoenthal 1989).

${ }^{8}$ Neben der ausführlichen Paarschreibung existieren auch verschiedene Kurzschreibungen, die in einigen Weiterentwicklungen eigenständige Alternativen zur Paarschreibung darstellen, z. B. Schreibungen mit dem statischen und dynamischen Unterstrich sowie mit Asterisk*, welche die Geschlechterdichotomie aufsprengen und für queere Geschlechterentwürfe in der Sprache Raum schaffen sollen (vgl. Hornscheidt 2011).

${ }^{9}$ Die Linguistik erstellt hierfür Korpora, die auch für Nicht-LinguistInnen zugänglich sind; besonders einschlägig ist das Corpus Search, Management and Analysis System COSMAS II des Instituts für deutsche Sprache in Mannheim, mit dem in derzeit 113 Korpora mit einem Schwerpunkt auf Tageszeitungen recherchiert werden kann, siehe: http://www.ids-mannheim.de/cosmas2/ [Stand: 20.03.2014].
} 
Personenreferenzform kann in der Regel eine semantische Rolle ermittelt werden; sie ergibt sich aus der Semantik desjenigen Verbs, mit dem die Personenreferenzform zusammen vorkommt. Für die Frage nach HandlungsträgerInnenschaft ist die semantische Rolle ,Agens“ (= HandelndeR, HandlungsträgerIn) besonders wichtig, z. B. Lena in Lena fährt mit dem Fahrrad den Berg hoch. Zum einen ermöglicht deren Kodierung die Abfrage, welches Geschlecht häufiger in der Agens-Rolle erscheint, zum anderen erlaubt die Analyse der Verben die Abfrage, welche Handlungsverben (im Beispiel: fahren) im Einzelnen für die Geschlechter charakteristisch sind. HandlungsträgerInnenschaft ist keine vage subjektive Analysekategorie, sondern mittels der linguistischen Klassifizierungen präzise fassbar. Die Kategorie der semantischen Rolle ermöglicht weiterhin quantitative Auswertungen dahingehend, welches Geschlecht eher von einer Handlung oder einem Vorgang betroffen ist (die für diese Auswertung relevante Kodierung unter der Kategorie der semantischen Rollen heißt ,Affiziertes Objekt' und ,Patiens') und welches bevorzugt als BesitzendeR (semantische Rolle ,PossessorIn') konzipiert ist (vgl. weiterführend zu den semantischen Rollen Primus 2012). Über die qualitative Analyse dessen, was im Einzelnen beispielsweise besessen wird, ergeben sich weitere aufschlussreiche Interpretationsansätze.

Das Schema berücksichtigt auch verdeckte Prädikationen, um gerade implizites Wissen herauszuarbeiten. Ein Fokus liegt auf attribuierten Nominalisierungen, im Einzelnen: auf sogenannten Genitivus subiectivus/obiectivus-Konstruktionen, bei denen die Personenreferenzform Genitivattribut zum nominalisierten Verb ist (z. B. Lisas Mitarbeit = ,Lisa arbeitet mit/hat mitgearbeitet'), und auf possessiven Nominalkonstruktionen (z. B. Cindys Fahrrad oder das Fahrrad von Cindy). Die Nominalkonstruktionen werden in Verbalkonstruktionen umgewandelt und, mit dem Zusatz Nominalkonstruktion versehen, als Verbalkonstruktion regelhaft kodiert.

Auf der Textebene dann interessieren Abbildungen im Schulbuch. Da in dieser Untersuchung die Konstruktionsleistung sprachlicher Zeichen im Mittelpunkt steht, werden keine systematischen Bildanalysen betrieben ${ }^{10}$, sondern Abbildungen vor allem dann in die Datenerhebung einbezogen, wenn über diese die Referenz vereindeutigt wird oder wenn Abbildungen von Personen Erwartetem entgegenarbeiten (z. B. die Abbildung von Tina, wie die Figur im Text genannt wird, die ohne jedes stereotype weibliche Attribut, dagegen androgyn mit Irokesenhaarschnitt und weiter Kleidung gezeichnet ist).

Die Teilergebnisse der Datenauswertung zeigen in der Zusammenschau dann, welche Konzepte von Mann und Frau in den Schulbüchern entworfen werden, was zum jeweiligen Konzept standardwertig dazuzugehören scheint und was eher als randständig gilt (vgl. auch MangasserWahl 2000).

\section{Untersuchungsergebnisse}

Im Folgenden werden ausgewählte Ergebnisse der Datenauswertung der 12 zeitgenössischen und vier historischen Mathematikbücher vorgestellt und Interpretationslinien entwickelt. Auf welches Standardpersonal wird in den Mathematikaufgaben nun zurückgegriffen und welche Geschlechterkonzeption konstituiert? Dieser Frage soll sich zunächst über ein traditionelles Verfahren angenähert werden: über das Zahlenverhältnis von weiblichen und männlichen Personenreferenzformen. Berücksichtigen wir nur jene Formen, mit denen dem Kotext nach

\footnotetext{
${ }^{10}$ Ein überzeugendes Beispiel für Bildanalysen von Schulbüchern findet sich bei Moser (2013).
} 
entweder eindeutig weibliche Personen oder männliche bezeichnet sein sollen, so fällt für die zeitgenössischen Mathematikbücher das Verhältnis beinahe gleichwertig mit leichtem MännerÜberhang aus; und auch, wenn wir jene maskulinen Formen in die Auswertung einbeziehen, die zwar sehr wahrscheinlich, dem Kotext nach aber nicht eindeutig männlich referieren, verschiebt sich das Zahlenverhältnis nur unwesentlich, allerdings weiter zugunsten der männlich referierenden Formen. In den historischen Rechenbüchern sind Personenreferenzformen für Frauen/Mädchen dagegen stark unterrepräsentiert: 1 weiblich referierende Personenreferenzform sieht sich in den kaiserzeitlichen Büchern 2 bis 9 männlichen Personenreferenzformen gegenüber; in den 1950er Jahren kommen auf 1 weibliche Form 1,5 bis 2 männliche. Im Laufe der Jahrzehnte hat sich das Zahlenverhältnis also deutlich angeglichen. Den beiden dominierenden Geschlechtern ist nun beinahe gleich viel Raum im Schulbuch gegeben.

Bislang war, nicht ohne Grund, lediglich von konventionalisiert gebrauchten Referenzformen für männliche und weibliche Personen die Rede. Die untersuchten Schulbücher scheinen ein ZweiGeschlechter-Modell auch nicht kritisch zu hinterfragen - in der deutschen Bildungspolitik steht es hingegen zur Debatte. In den vergangenen zehn Jahren wurden immer wieder Forderungen laut und zuletzt anlässlich des neuen Bildungsplankonzepts des Landes Baden-Württemberg hitzige Diskussionen darüber geführt, ob bzw. dass sexuelle Vielfalt und alternative Geschlechter- und auch Familienentwürfe in allen Schulfächern und Lehr-/Lernmaterialien thematisiert werden und selbstverständlich präsent sein sollen. Die gegenwärtig eingesetzten Mathematikbücher bieten kaum Interpretationsangebote, die das heterosexuelle ZweiGeschlechter-Modell überschreiten würden; in während der Kaiserzeit und der Adenauer-Ära entstandenen Schulbüchern ist eine Kritik am Binarismus erst recht nicht zu erwarten und auch empirisch nicht zu belegen. Bis heute tauchen also weder gleichgeschlechtliche Eltern in einer Aufgabenstellung auf noch kommen explizit als solche kenntlich gemachte alleinerziehende Väter/Mütter oder Scheidungskinder vor. Wird in den Büchern näher auf die einzelnen Mitglieder einer Familie eingegangen, besteht diese immer aus einem Vater und einer Mutter sowie ein oder zwei Kindern. Einzig fallen aus dieser Matrix im zeitgenössischen Mathematikbuch die deutlich androgyne Abbildung von Tina sowie einige Unisex-Vornamen wie Alex, Chris, Sasha. Nach Auskunft von SchulbuchautorInnen sei mit diesen Vornamen jedoch kein Einbezug queerer Geschlechterentwürfe intendiert, sondern wolle der Vornamenrealität im Klassenzimmer entsprochen werden. Im Mathematikbuch sind die bildungspolitischen Bestrebungen bislang also nicht angekommen.

Die beinahe paritätische Verteilung weiblicher und männlicher Personenreferenzformen im zeitgenössischen Mathematikbuch legt nahe, dass beispielsweise Berufszuweisungen, Aktivität sowie Besitztümer ebenfalls gleichmäßig auf die Geschlechter verteilt sein müssten. Die Berufsbezeichnungen wollen diese Erwartung nicht erfüllen, hier wird über die drei Zeitschnitte eine Tradition fortgeschrieben: In Vergangenheit und Gegenwart bezeichnen diese Ausdrücke mehr als doppelt so häufig eindeutig Männer und bieten den männlichen SchulbuchbenutzerInnen ein sehr viel größeres Repertoire an unterschiedlichen Berufen und Identifikationsmöglichkeiten - es reicht vom Bauern/Landwirt über den Gemüse- oder Möbelhändler und den in den neueren Büchern hinzugekommenen Skispringer bis zum Firmenchef. Die Tätigkeitsfelder für Männer verändern sich dabei im Lauf der Zeit: Stehen die Berufsbezeichnungen in den kaiserzeitlichen Büchern schwerpunktmäßig für einfache ArbeiterTätigkeiten und hat auch das Militär seinen festen Platz, so liegt in den 1950er Jahren ein stärkerer Fokus auf dem Handwerk sowie dem Klein- und Großhandel. In allen Jahrzehnten typische, schon in den historischen Rechenbüchern auftauchende Frauenberufsbezeichnungen 
sind die Verkäuferin - heute vor allem die Verkäuferin von Kleidung, in der Adenauer-Zeit und zuvor vor allem die Lebensmittelverkäuferin - sowie die mit Lebensmitteln, z. T. auch Luxusartikeln Handel treibende Frau und die Lehrerin; letztere hat einen festen Platz im zeitgenössischen Mathematikbuch, den sie sich allerdings nach einer kurzen Evidenz für die kaiserzeitlichen Jahre nach in dieser Hinsicht reaktionären 1950er Jahren erst zurückerobern musste und festigen konnte. Hausfrauen und Bäuerinnen dagegen finden sich im heutigen Mathematikbuch nicht mehr, wobei die in der Landwirtschaft als berufstätig ausgewiesenen Frauen sowohl in den Heften und Büchern der 1890er Jahren wie auch in den 1950er Jahren hier noch eklatanter - im Vergleich zu den Männern, den Bauern, quantitativ sehr schwach in Erscheinung treten. Nachdem im zeitgenössischen Mathematikbuch Berufsbezeichnungen ohnehin im Vergleich zu den übrigen Personenreferenzformen seltener vorkommen ${ }^{11}$, ist die Datengrundlage mit insgesamt 16 unterschiedlichen Berufsbezeichnungen für Frauen sehr gering und eine weiterführende Interpretation nur bedingt möglich. Doch im Kontrast $\mathrm{zu}$ den Männerberufsbezeichnungen ergeben sich interessante Befunde: Technische Berufe (Landvermesser, Konstrukteur) und solche, die schwere körperliche Tätigkeit erfordern (Tischler, Installateur, Dachdecker), sind etwas für Männer, sogenanntes leichtes Handwerk (Goldschmiedin, Konditorin) ausschließlich etwas für Frauen; Unternehmertum und Führungspositionen (Direktor, Chef, Geschäftsführer, Abteilungsleiter) sind männlich dominiert, doch findet sich im Datenmaterial auch eine Schulleiterin - der Kontext Schule als typisch weibliches Betätigungsfeld jedoch relativiert diesen Befund. In den wilhelminischen Rechenbüchern konzentrieren sich die Berufsbezeichnungen für Frauen auf den Landwirtschaftsund Hauswirtschaftssektor, wobei beispielsweise mit Personenreferenzformen wie Bauersfrau (siehe auch für den Handelssektor: Kaufmannsfrau) bereits auf Ebene der Wortbildung eine Abhängigkeit bzw. Nachgeordnetheit der Frau vom bzw. zum Mann ausgedrückt wird; es ist dabei diskutabel, ob es sich bei den genannten Beispielen um Berufsbezeichnungen handelt (Bauersfrau = ,weiblicher Bauer", im Sinn von: als Bäuerin berufstätige Frau) oder eher um den Ausdruck einer matriomoniellen Zugehörigkeit (Bauersfrau =, des Bauers (Ehe-)Frau', im Sinn von: die Frau als Pendant zum Mann, der ein Bauer ist). Der Kotext schafft hier in den meisten Fällen Klarheit und erlaubt, den so bezeichneten Figuren den Status einer Berufstätigkeit zuzuweisen. In Leitungsfunktion kommen Frauen in der Vergangenheit nicht vor, einzig die Lehrerin ist Beleg für eine höhere Bildung erfordernde Tätigkeit von Frauen.

Selten durchkreuzen die auftauchenden Berufsbezeichnungen stereotype Zuweisungen; wenn, dann allerdings lediglich in eine Richtung: Wir können heute von einer Rennfahrerin lesen, finden aber keine Inanspruchnahme sogenannter Frauenberufe unter den männlichen Berufsbezeichnungen. Die Geschlechterstereotypik scheint nur in eine Richtung durchlässig zu sein, was Berufsdomänen von Frauen wenig attraktiv und prestigeträchtig erscheinen lässt bzw. diese Wertung unterstützt.

Ausdrücke, die ein Verwandtschaftsverhältnis anzeigen, verorten Personen in der Sphäre Familie. Bereits ein quantitativer Zugriff auf diese Formen, getrennt in Bezeichnungen für Männer/Jungen und Frauen/Mädchen, verschafft einen ersten Eindruck, welches Geschlecht

\footnotetext{
${ }^{11}$ Vornamen dagegen kommen deutlich häufiger im zeitgenössischen Mathematikbuch vor als noch in den 1950er Jahren; zudem sind Rechenoperationen im Vergleich zu den historischen Rechenbüchern weitaus seltener im Stil klassischer Sachaufgaben mit abstraktem Personal nach dem Muster Ein Kaufmann erwirbt... formuliert. Es überwiegen heute zunehmend direkte Handlungsaufforderungen an die SchulbuchbenutzerInnen und es besteht das Schulbuchpersonal hauptsächlich aus gleichaltrigen Identifikationsfiguren, die, mit einem Vornamen bezeichnet, individualisierter gestaltet sind. Beide Befunde sind nach Angaben der SchulbuchmacherInnen in der stärkeren SchülerInnen-Orientierung von Unterricht und der Autonomisierung von Lernprozessen begründet.
} 
bevorzugt in familiären Bezügen erscheint. Die Verwandtschaftsbezeichnungen sind in den Mathematikbüchern zahlenmäßig gleichmäßig auf die zwei Geschlechter verteilt ${ }^{12}$, was dem Stereotyp der Frau als Familienmensch entgegenarbeitet; Männer sind damit ebenso häufig - in den kaiserzeitlichen Rechenbüchern sogar tendenziell häufiger - in ihrer Familienrolle expliziert. Ein anfänglicher Befund mit kleinerem Untersuchungskorpus an zeitgenössischen Mathematikbüchern, wonach Mutter signifikant häufiger als Vater im Belegmaterial vorzufinden war (vgl. Ott 2014a), bestätigte sich im vorliegenden größeren Korpus nicht. Auch die Auswertung der possessiven Genitiv- und Präpositionalphrasen (ihre Mutter, die Tante von Lucas) zeigte, dass verwandtschaftliche Verhältnisse im Allgemeinen nicht überzufällig ein Geschlecht charakterisieren. Doch ergibt sich auf den zweiten Blick wieder ein differenzierteres Bild: Es wird ausschließlich Mutter in einigen Fällen ohne Artikelwort verwendet, z. B. Mutter sagt zu Karin ... oder Mutter kauft 20 Kilo Äpfel anstelle von Die Mutter sagt zu Karin ... und Die Mutter/Ihre Mutter kauft 20 Kilo Äpfel. Die Verwandtschaftsbezeichnung Mutter nähert sich in dieser Verwendungsweise von der Klasse der Gattungsnamen an die Klasse der Eigennamen an. Mit dieser terminologischen Differenzierungsmöglichkeit wird auch eine funktionale Verschiebung beschreibbar: Eine Person wird so eng in Bezug auf ihre familiäre Rolle gedacht, dass diese Rolle namengebend werden kann und die personale Individualität, die besonders deutlich mit einem ,echten' Eigennamen versprachlicht wäre, in den Hintergrund tritt. Mutter in der Verwendung eines Eigennamens stellt dabei gleichzeitig eine Intimisierung dar, weil derartige Verwendungsweisen vornehmlich im privaten Sprachgebrauch anzusiedeln sind.

Kaufhandlungen waren und sind beliebte Rahmensituationen in mathematischen Sachaufgaben; für die Analyse auf Geschlechterkonzepte ist von Interesse, ob hier Auffälligkeiten in deren Verteilung auf die Geschlechter festzustellen sind. Über die quantitativ-qualitative Auswertung von Verben, die ein Kaufgeschäft aus KäuferInnen-Perspektive bezeichnen, sowie der damit relationierten Einheiten, erhalten wir Aufschluss darüber, ob im Schulbuch bestimmte Kaufobjekte bei einem Geschlecht prominenter vertreten sind als bei dem anderen. In historischen Mathematikbüchern kauft das weibliche Personal überwiegend Lebensmittel und Textilien für die private Weiterverarbeitung, in den 1950er Jahren kommen Küchenutensilien und kleinere Haushaltsgeräte dazu. Wenn männliche Personen Lebensmittel, Haushaltswaren oder Textilien käuflich erwerben, dann fast immer gewerblich: So kauft der Kaufmann Kaffeemengen zum Weiterverkauf in seinem Geschäft. An der Oberfläche sind die Kaufgegenstände zum Teil ähnlich, mit Blick auf die konkreten Figuren, die etwas kaufen, wird rasch der meist unterschiedliche Kotext der Kaufhandlung (privat versus gewerblich) deutlich. Geld- und Wertpapiergeschäfte sind zudem Hoheitsgebiet der Männer oder selten auch unverheirateter Frauen (Fräulein). Diese funktionale Trennung in gewerbliche männliche Kaufhandlungen und die Familienarbeit betreffende weibliche Kaufhandlungen fällt in den Adenauer-Rechenbüchern nicht mehr ganz so starr aus; darin treten dann auch Männer auf, die größere Anschaffungen für den Haushalt tätigen. Aktuelle Mathematikbücher scheinen diese Trennung bewusst nicht weiterschreiben zu wollen. So sind elektronische Geräte, wie Computer und Musikabspielgeräte, sowohl bei Mädchen/Frauen als auch bei den Jungen/Männern beliebt. Bei den Kaufhandlungen finden sich Belege, die traditionelle Rollenzuweisungen durchkreuzen: Unter anderem kaufen sich Mädchen ein Mofa oder eine Bohrmaschine und erwerben Männer - wie schon in den 1950er Jahren angelegt - Haushaltsgeräte, z. B. eine Waschmaschine oder einen Geschirrspüler. Wird diese Perspektive in einem nächsten Schritt auf Besitztümer generell ausgeweitet und

\footnotetext{
${ }^{12}$ Das Feld der Verwandtschaftsbezeichnung ist weitgehend binär und heteronormativ strukturiert: Mutter - Vater, Schwester-Bruder, Oma-Opa, Tante-Onkel.
} 
werden Possessivzuschreibungen und jene Einheiten mitgewertet, welche mit Personenreferenzformen relationiert sind, die unter der Kategorie ,Semantische Rolle" als ,PossessorIn" kodiert sind, so sticht besonders heraus: Haus und Grundbesitz ist im Mathematikbuch in der Hand der Männer. Zeitgenössische Mathematikbücher führen hierin eine Tradition fort, die mindestens bis in die Kaiserzeit zurückreicht, wenngleich der Befund für historische Rechenbücher noch eklatanter ausfällt. Die Eigen-Vorgabe der SchulbuchmacherInnen, stereotype Zuschreibungen in der gegenwärtigen Schulbucharbeit zu vermeiden, kann somit nur bedingt als erfolgreich umgesetzt bezeichnet werden. Dieser komplexe Befund steht vielleicht auch symptomatisch für den Mangel an linguistischer Forschung, die deutlich machen könnte, auf welche ganz unterschiedliche Weise Sprache am Aufbau von Geschlechterkonzepten mitarbeitet. Es ist augenscheinlich nicht damit getan, bestimmte Verben und Objekttypen möglichst paritätisch auf männliche und weibliche Personenreferenzformen zu verteilen.

Zum Abschluss der exemplarischen Ergebnispräsentation sei der isolierte Blick auf zeitgenössische Mathematikbücher gestattet, um in aller Kürze einen Vergleich zu weiteren Prämissen der SchulbuchmacherInnen ziehen $\mathrm{zu}$ können. Insgesamt zeigt sich bei der Auswertung der semantischen Verbklassen ein lediglich leichtes Ungleichgewicht in der Verteilung von Handlungsverben auf Personenreferenzformen für Frauen/Mädchen und Männer/Jungen, und zwar insofern, als etwas mehr männlich referierende Formen in der semantischen Rolle ,Agens' zusammen mit einem Handlungsverb vorkommen. Damit ist Aktivität weitgehend nicht geschlechtsstereotyp gezeichnet. Vordergründig lösen zeitgenössische Mathematikbücher auf dieser Analyseebene ein, was die InterviewpartnerInnen als Maßgabe für Geschlechtersensibilität im Schulbuch genannt hatten. Doch die Gesamtauswertung der untersuchten zeitgenössischen Bücher täuscht Gleichmäßigkeiten vor, die in einer Einzelbetrachtung der Schulbücher nicht zu bestätigen sind: Tatsächlich fallen die Zahlenverhältnisse je nach Schulbuch sehr unterschiedlich aus. In einigen ist ein leichtes Übergewicht weiblicher HandlungsträgerInnenschaft festzustellen, in anderen Schulbüchern findet sich doppelt so häufig eine Verbindung aus männlicher Personenreferenzform und Handlungsverb. GutachterInnen scheint Letzteres nicht aufgefallen $\mathrm{zu}$ sein oder eine von GutachterInnen geäußerte Kritik daran veranlasste die Zulassungsbehörde nicht, vom Verlag eine Überarbeitung einzufordern.

\section{Zusammenfassung}

Das Medium der schulischen Bildung, das Schulbuch, hat vor allem durch die Schulbuchkritik der 1970er Jahre größere Änderungen erfahren. Auch der Umgang mit der Identitätskategorie Geschlecht hat sich dadurch gewandelt. An diesem Ausschnitt des Bildungswesens wurde das Verhältnis von Bildung und Differenz exemplarisch nachvollzogen und es wurde untersucht, welche Geschlechterkonzepte im und mit dem Schulbuch (re-)produziert wurden bzw. werden und inwiefern sich AkteurInnen der Schulbucharbeit in der jüngeren Vergangenheit an dieser Wissensproduktion beteiligt haben. Zunächst einmal ist festzuhalten: Es hat sich Einiges im Schulbuch hin zu einem emanzipierteren Männer- und Frauenbild geändert. Junge Männer backen heute im Mathematikbuch Kuchen und haben Liebeskummer, d. h. sie dürfen Gefühle zulassen; junge Frauen kaufen Bohrmaschinen und bekommen auch schon einmal Nobelpreisträgerinnen als Vorbild genannt. Doch bereits durch die oben vorgestellten 
Teilauswertungen wurde deutlich, dass vor allem bei einer differenzierteren Betrachtung der Schulbuchsprache Problemstellen identifiziert werden können. ${ }^{13}$ Zuvorderst ist die Beschränkung auf das heterosexuelle Zwei-Geschlechter-Modell zu nennen, das weitere Geschlechter- und Sexualitätsentwürfe von dem nicht erklärungsbedürftigen Zwei-GeschlechterModell separiert. Zum zweiten weist Berufstätigkeit im Mathematikbuch seit jeher und weiterhin eine männliche Kodierung auf und liefert damit unterschwellig Vorstellungen des Mannes als Hauptverdiener Vorschub. Begünstigt wird dies noch dadurch, dass in einigen aktuellen Mathematikbüchern ausschließlich am Beispiel weiblicher Schulbuchfiguren das Thema Teilzeitarbeit und Stundenreduktion angesprochen wird und ausschließlich männliche Figuren Gehaltserhöhungen erhalten. Für junge Mädchen bieten diese Medien insgesamt eine nur sehr beschränkte Auswahl an berufstätigen Identifikationsfiguren, selbst sogenannte Frauenberufe aus dem Erziehungs- und Pflegebereich bleiben weitgehend ungenannt. Familienarbeit dagegen ist in der Schulbuchwelt auf Frauen und Männer gleichmäßig verteilt, doch gerade in Bezug auf die Mutterrolle ist im Schulbuch ein Nähekonzept angelegt, das beim männlichen Familienmenschen so nicht vorzufinden ist. Mit Einschränkungen im Bereich Familie und Haushalt dringen außerdem sehr viel wahrscheinlicher weibliche Personen in typisch männliche Domänen vor als umgekehrt. Undoing gender ist somit eher dem weiblichen Schulbuchpersonal möglich.

Unter Berücksichtigung aller Teilergebnisse der Auswertung drängt sich weiterhin folgender Schluss auf: SchulbuchmacherInnen orientieren sich bei der Konstruktion der Schulbuchwelt weniger am Gleichheitsideal als an einer Vorstellung sozialer Realität. Sie laufen darin Gefahr, Geschlechterstereotype zu verfestigen und im Sinn eines doing gender als doing difference zu reproduzieren - vor allem in Bezug auf Berufstätigkeit und, wie oben ausgeführt, zum Teil auf sehr subtile Weise.

Den Interviews nach sollte die Einflussnahme von normsetzenden Instanzen auf die Umsetzung der Gleichberechtigung der Geschlechter im Schulbuch auch nicht überbewertet werden. Es scheint sich in der Schulbucharbeit und Zulassungspraxis in den vergangenen 15 bis 20 Jahren ein gewisser Standard entwickelt zu haben, der stets zwischen dem Anspruch eines gesellschaftlichen Ideals und dem, was als soziale Realität empfunden wird, der man mit geschlechtsspezifischen Identifikationsangeboten entsprechen möchte, pendelt. Frei von Klischees und Geschlechterstereotypie ist dieser Standard nicht.

Ein schärferer Blick, so sollte gezeigt werden, auf das Sprachmaterial, das an der Konstruktion solcher stereotyper oder nicht-stereotyper Geschlechterkonzepte beteiligt ist, macht eine differenzierte Beschreibung der einzelnen konzeptuellen Bausteine möglich. Wenngleich diese Analyse freilich ebenfalls nicht gänzlich hinter das zu untersuchende Material zurück kann, so wird durch die kategorialen Feindifferenzierungen doch eine größere Objektivität erreicht beim Aufspüren von Stereotypen, indem diese nämlich nicht von vornherein die Analyse und Datenerhebung leiten, sondern Stereotype erst in der Gesamtzusammenschau der Sprachdatenauswertungen als mögliche Interpretationsschablonen einbezogen werden. Gerade bei (bildungs-)historischen Untersuchungen zu sprachlichen Praktiken des doing und undoing gender ist ein solches Vorgehen anzuraten. Denn es sensibilisiert die/den ForscherIn, den Untersuchungsgegenstand nicht mit einer von gegenwärtigen Perspektiven auf das historische Material geleiteten zu starken inhaltlichen Vorstrukturierung anzugehen. Insofern will die vorgestellte Schulbuchanalyse auch ein Methodenbeitrag zur bildungshistorischen Geschlechterforschung sein.

${ }^{13} \mathrm{Zu}$ Ergebnissen thematisch verwandter neuerer Studien vgl. Schärer (2008); Bittner (2012); Moser (2013). 


\section{Quellen- und Literaturverzeichnis}

\section{Untersuchungskorpus/Quellen}

Backhaus, K. und Wiese, B. (1899). Rechenbuch für mehrklassige Volksschulen. Siebentes Heft. 3. Auflage. Bielefeld und Leipzig.

Bauhoff, E. und Wynands, A. (Hrsg.) (2005). Mathe aktiv M7. Mathematisches Unterrichtswerk für Hauptschulen. Braunschweig.

Böer, H. u. a. (2007). mathe live 7. Mathematik für Sekundarstufe I. Stuttgart.

Englmaier, C. u. a. (2011). Mathematik 7 II/III. Realschule Bayern. Braunschweig.

Eyselein, W. und Hagen, M. (ca. 1957). Wir rechnen. Rechenbuch für Volksschulen. 7. Schülerjahrgang. Bamberg, Freising und Würzburg.

Greulich, D. u. a. (2007). Lambacher Schweizer 7. Mathematik für Gymnasien. NordrheinWestfalen. Stuttgart.

Heuner, J. F. (1890). Aufgaben zum Kopf-und Ziffernrechnen. Schüler-Ausgabe für Landschulen. Heft C. Oberklasse. 159. Auflage. Ansbach.

Kleine, M. und Ludwig, M. (Hrsg.) (2012). Mathe.Logo 7. Sekundarstufe I. Bamberg.

Kleine, M., Ludwig, M. und Weixler, P. (Hrsg.) (2012). Mathe.Logo 7 II. Realschule Bayern. Bamberg.

Meins, H. (1955). Hamburger Rechenbuch für das 7. Schuljahr. Hamburg.

Reich, G. und Rothmeier, G. (Hrsg.) (2003). Thema Mathe - neu 7/II. Mathematik für sechsstufige Realschulen. [Bayern.] Bamberg.

Sailer, W., Vollath, E. und Weidner, S. (Hrsg.) (2011). Formel 7. Mathematik. Bamberg.

Sailer, W., Vollath, E. und Weidner, S. (Hrsg.) (2004). Formel 7. Mathematik für Hauptschulen einschließlich Mittlere-Reife-Zug. Bamberg.

Schätz, U. und Eisentraut, F. (Hrsg.) (2009). delta - neu 7. Mathematik für Gymnasien. [Bayern.] Bamberg.

Schätz, U. und Eisentraut, F. (Hrsg.) (2005). delta 7. Mathematik für Gymnasien. [Bayern.] 2. Auflage. Bamberg.

Schmid, A. u. a. (2005). Lambacher Schweizer 7. Mathematik für Gymnasien. Bayern. Stuttgart. 


\section{Literatur}

Ballis, A. und Gaebert, D.-K. (2012). Lehr- und Lernmedien als Kristallisationspunkte der Literaturdidaktik. Ergebnisse einer Interviewstudie. In: Ballis, A. und Peyer, A. (Hrsg.). Lernmedien und Lernaufgaben im Deutschunterricht. Konzeptionen und Analysen. Bad Heilbrunn, 135-150.

Bayerisches Staatsministerium für Bildung und Kultus, Wissenschaft und Kunst [StMUKWK] (2014). Allgemeiner Kriterienkatalog zur Beurteilung von Lernmitteln. http://www.km.bayern.de/download/7432_allgemeiner_kriterienkatalog_januar_2014.pdf (letzter Zugriff: 10.03.2014).

Berger, P. L. und Luckmann, T. (1977 [engl. 1966]). Die gesellschaftliche Konstruktion der Wirklichkeit. Eine Theorie der Wissenssoziologie. 5. Auflage. Frankfurt am Main.

Bittner, M. (2012). Geschlechterkonstruktionen und die Darstellung von Lesben, Schwulen, Bisexuellen, Trans* und Inter* (LSBTI) in Schulbüchern. Eine gleichstellungsorientierte Analyse. Max-Traeger-Stiftung, Frankfurt am Main.

Brieske, R. (2007). Schulbucheinsatz. Zum Umgang mit einem Leit(d)medium. In: Praxis Geschichte 3, 29-32.

Fichera, U. (1996). Die Schulbuchdiskussion in der BRD - Beiträge zur Neugestaltung des Geschlechterverhältnisses. Bestandsaufnahme und Sekundäranalyse. Frankfurt am Main.

Heer, N. (2011). Aufgaben im Schulbuch aus textlinguistischer Sicht. Zur intra- und intertextuellen Orientierung. In: Matthes, E. und Schütze, S. (Hrsg.). Aufgaben im Schulbuch. Bad Heilbrunn, 43-56.

Hellinger, M. und Bierbach, C. (1993). Eine Sprache für beide Geschlechter. Richtlinien für einen nicht-sexistischen Sprachgebrauch. Deutsche UNESCO-Kommission, Bonn. http://www.unesco.de/fileadmin/medien/Dokumente/Bibliothek/eine_sprache.pdf (letzter Zugriff: 10.03.2014).

Höhne, T. (2003). Schulbuchwissen. Umrisse einer Wissens- und Medientheorie des Schulbuchs. Dissertation, Johann-Wolfgang-Goethe-Universität. Frankfurt am Main.

Hornscheidt, L. (2012). feministische w_orte. ein lern-, denk- und handlungsbuch zu sprache und diskriminierung, gender studies und feministischer linguistik. Frankfurt am Main.

Hornscheidt, L. (2011). Dyke_Trans schreiben lernen. Schreiben als feministische Praxis. In: AK Feministische Sprachpraxis (Hrsg.). Feminismus schreiben lernen. Frankfurt am Main, 100-138. 
Hunze, A. (2003). Geschlechtertypisierung in Schulbüchern. In: Stürzer, M. u.a. (Hrsg.). Geschlechterverhältnisse in der Schule. Opladen, 53-82.

Lässig, S. (2009). Textbooks and Beyond: Educational Media in Context(s). The Journal of Educational Media, Memory, and Science 1 (1), 1-20.

Leppek, S. (2002). Die Zulassung und Einführung von Schulbüchern und anderen Lernmitteln an staatlichen deutschen Schulen. Verfassungs- und verwaltungsrechtliche Grundfragen. Marburg.

Lopatecki, C. und Lüking, I. (1989). Bescheiden, sittsam und rein? Rollenklischees in Mathematik-Schulbüchern für die Sekundarstufe I. Bremen.

Mangasser-Wahl, M. (2000). Von der Prototypentheorie zur empirischen Semantik. Dargestellt am Beispiel von Frauenkategorisierungen. Frankfurt am Main.

Moser, F. (2013). Social Construction of Gender-(un)fairness. An Analysis of Educational Material and Individual Language Use. Dissertation. Freie Universität Berlin.

Müller, G. (1997). Beschränkungen für Binomialbildug im Deutschen. Ein Beitrag zur Interaktion von Phraseologie und Grammatik. In: Zeitschrift für Sprachwissenschaft 16(1/2), 551 .

Neumann, D. (2015): Bildungsmedien Online. Kostenloses Lehrmaterial aus dem Internet: Marktsichtung und empirische Nutzungsanalyse. Bad Heilbrunn.

Ott, C. (2014a). Geschlechtsidentität(en) im Mathebuch. Was die Sprache in Bildungsmedien über ihre Gesellschaft verrät. In: Theurer, C., Siedenbiedel, C. und Budde, J. (Hrsg.). Lernen und Geschlecht. Immenhausen, 241-254.

Ott, C. (2014b). Das Schulbuch beim Wort nehmen. Linguistische Methodik in der Schulbuchforschung. In: Knecht, P., Aamotsbakken, B., Matthes, E. und Schütze, S. (Hrsg.). Methodologie und Methoden der Schulbuch- und Lehrmittelforschung. Bad Heilbrunn, 254-263.

Postupa, J. und Weth, T. (2011). Mathematikaufgaben im Spiegel ihrer Zeit. In: Matthes, E. und Schütze, S. (Hrsg.). Aufgaben im Schulbuch. Bad Heilbrunn, 165-182.

Primus, B. (2012). Semantische Rollen. Heidelberg.

Schärer, F. (2008). Père, mère, roi et sorcière. La représentation des deux sexes et de la catégorie du genre dans les manuels scolaires de l'école primaire de la Suisse alémanique et de la Suisse romande. Bern.

Schoenthal, G. (1989). Personenbezeichnungen im Deutschen als Gegenstand feministischer Sprachkritik. In: Zeitschrift für germanistische Linguistik 17, 296-314. 
Spieß, C. (2012). Linguistische Genderforschung und Diskurslinguistik. Theorie - Methode Praxis. In: Günthner, S., Hüpper, D. und Spieß, C. (Hrsg.). Genderlinguistik. Sprachliche Konstruktionen von Geschlechtsidentität. Berlin und Boston, 53-85.

Stein, G. (1979). Immer Ärger mit den Schulbüchern. Ein Beitrag zum Verhältnis zwischen Pädagogik und Politik. Dokumentarischer Teil. Stuttgart.

Stöber, G. (2010). Schulbuchzulassung in Deutschland. Grundlagen, Verfahrensweisen und Diskussionen. Eckert. Beiträge 3. http://d-nb.info/1002260256/34 (letzter Zugriff: 10.03.2014).

Verband Bildungsmedien e.V. (Hrsg.) (2013). Bisherige Ergebnisse des Forschungsprojekts „Bildungsmedien Online“ der Universität Augsburg. Lehrerbefragung „Kostenlose Bildungsmedien online“. Abstract IV. http://www.bildungsmedien.de/presse/pressedownloads/forschungsprojekt-augsburg (letzter Zugriff: 10.03.2014).

Warnke, I. H. (2013). Diskurs als Praxis und Arrangement - Zum Status von Konstruktion und Repräsentation in der Diskurslinguistik. In: Viehöver, W., Keller, R. und Schneider, W. (Hrsg.). Diskurs - Sprache - Wissen. Interdisziplinäre Beiträge zum Verhältnis von Sprache und Wissen in der Diskursforschung. Wiesbaden, 97-118.

Wendt, P. (2010). Schulbuchzulassung: Verfahrensänderung oder Verzicht auf Zulassungsverfahren? In: Fuchs, E., Kahlert, J. und Sandfuchs, U. (Hrsg.). Schulbuch konkret: Kontexte - Produktion - Unterricht. Bad Heilbrunn, 83-96. 